\title{
Video Article \\ Assessment of Respiratory Function in Conscious Mice by Double-chamber Plethysmography
}

\author{
Samuel Mailhot-Larouche ${ }^{1}$, Louis Deschênes ${ }^{1}$, Katherine Lortie ${ }^{1}$, Morgan Gazzola ${ }^{1}$, David Marsolais ${ }^{1}$, David Brunet ${ }^{2}$, Annette Robichaud ${ }^{2}$, \\ Ynuk Bossé ${ }^{1}$ \\ ${ }^{1}$ Institut Universitaire de Cardiologie et de Pneumologie de Québec, Université Laval \\ ${ }^{2}$ SCIREQ Scientific Respiratory Equipment Inc.
}

Correspondence to: Annette Robichaud at Annette.Robichaud@scireq.com

URL: https://www.jove.com/video/57778

DOI: doi: $10.3791 / 57778$

Keywords: Medicine, Issue 137, Plethysmography, respiratory function, ventilation parameters, specific airway resistance, flow at midexpiratory volume, forced oscillation technique

Date Published: 7/10/2018

Citation: Mailhot-Larouche, S., Deschênes, L., Lortie, K., Gazzola, M., Marsolais, D., Brunet, D., Robichaud, A., Bossé, Y. Assessment of Respiratory Function in Conscious Mice by Double-chamber Plethysmography. J. Vis. Exp. (137), e57778, doi:10.3791/57778 (2018).

\section{Abstract}

Air volume changes created by a conscious subject breathing spontaneously within a body box are at the basis of plethysmography, a technique used to non-invasively assess some features of the respiratory function in humans as well as in laboratory animals. The present article focuses on the application of the double-chamber plethysmography (DCP) in small animals. It provides background information on the methodology as well as a detailed step-by-step procedure to successfully assess respiratory function in conscious, spontaneously breathing animals in a non-invasive manner. The DCP can be used to monitor the respiratory function of multiple animals in parallel, as well as to identify changes induced by aerosolized substances over a chosen time period and in a repeated manner. Experiments on control and allergic mice are used herein to demonstrate the utility of the technique, explain the associated outcome parameters, as well as to discuss the related advantages and shortcomings. Overall, the DCP provides valid and theoretically sound readouts that can be trusted to evaluate the respiratory function of conscious small animals both at baseline and after challenges with aerosolized substances.

\section{Video Link}

The video component of this article can be found at https://www.jove.com/video/57778/

\section{Introduction}

The increasing use of small animals to model human respiratory diseases has urged the development of techniques to quantitatively assess the functions of the respiratory system in those animals. Currently, the forced oscillation technique (FOT) is recognized as the most precise approach to assess respiratory mechanics in small animals ${ }^{1,2}$. However, as stated by the phenotyping uncertainty principle, what is gained in measurement precision with the FOT is traded off against a loss in noninvasiveness ${ }^{3}$. Indeed, FOT measurements are acquired under highly controlled experimental conditions that necessitate anesthesia, tracheotomy or oral intubation, as well as mechanical ventilation; a scenario far from real-life.

In situations where the experimental requirements proscribe the use of anesthetic agents or call for little or no deviation from the animal's natural physiological state, double-chamber plethysmography (DCP) can be considered. As its name indicates, a DCP setup consists of two connected rigid chambers built to isolate as hermetically as possible the animal's head (or nose), in the front chamber, from its thorax, in the rear chamber. Within the setup, the animal is conscious and breathes spontaneously while being restrained. Because the walls of the chambers cannot expand or retract, the motion of air in-and-out of the animal generates a corresponding but opposite waveform inside the rear chamber, as a result of the compression/decompression of surrounding air. The waveform due to nasal flow in the front chamber and the one related to the thoracic motion in the rear chamber can thus be separated and captured simultaneously. Depending on the design of the DCP setup, these waveforms can be acquired using a set of either pressure transducers or pneumotachographs to respectively record the changes in chamber pressure or airflow inand-out of the chambers as a function of time. The latter approach is more common nowadays.

While the animal's breathing frequency can be accurately determined by any kind of plethysmography techniques, the situation is not the same for the determination of tidal volume and its related ventilation parameters (e.g., minute ventilation, expiratory volume, etc.). As opposed to the whole-body plethysmograph (WBP) technique, where the animal's tidal volume is estimated from the box signal ${ }^{4,5}$, the DCP technique provides accurate assessments of tidal volume. This is related to the direct acquisition of the animal's thoracic movement in the rear chamber, which are proportional to the changes in lung volume during breathing.

In addition to these accurate ventilatory parameters (e.g., tidal volume, breathing frequency, and minute ventilation), some disturbances in the shape of the respiratory cycle can also be used to investigate neuronal aspects that govern the respiratory drive or respiratory reflexes. A specific example of that application would be the evaluation of the irritation potential of inhaled substances on the upper airway sensory neurons ${ }^{6}$. Here, the duration of a pause at the onset of expiration is determined using a parameter called end-inspiratory pause (EIP), also 
referred to as duration of braking ${ }^{6}$. The prolongation of this pause by an irritant substance is associated with the closure of the animal's glottis, causing a measurable period of braking in the first part of the expiration ${ }^{6,7}$.

Another important advantage of the DCP is that it provides two validated and undisputed parameters that are sensitive to airflow obstruction. One is called the flow at mid-tidal expiratory volume and is abbreviated EF50, 50,10 . It is the airflow at midway volume of each tidal breath during expiration. EF50 is extracted from the thoracic flow trace and can thus be measured without the front chamber (i.e., in a head-out configuration). The other one is called specific airway resistance and is abbreviated sRaw ${ }^{11,12,13}$. The determination of sRaw requires the simultaneous recording of the animal's nasal and thoracic flows as it is computed from the time delay between these separate respiratory traces at the point of zero flow at the end of inspiration. The rationale that describes the basis by which this delay relates to sRaw was expatiated previously ${ }^{11}$. Put simply, the changes in lung volume precede the air movement since a pressure gradient needs to develop in order to drive airflow. In a healthy animal breathing quietly, this delay is typically very small. However, the pressure gradient that is required to accommodate a given flow (e.g., a flow sufficient to provide adequate ventilation) is influenced by the degree of airway resistance. During bronchoconstriction, for example, the gradient of pressure needed to accommodate a given flow is greater, which implies that the animal has to work harder for breathing. A greater gradient of pressure in the animal's thorax also implies that a greater portion of the flow in-and-out of the rear chamber is due to decompression/ compression of air within the thorax, which is the portion of the total thoracic expansion/retraction that is out of phase with the nasal flow. The increased resistance due to bronchoconstriction will thus increase the delay between the rear and the front chambers and thereby increases sRaw. The gradient of pressure that drives airflow in-and-out of the lung is also influenced by the initial thoracic gas volume (TGV). At a greater TGV for example, the expansion/retraction of the thorax needed to generate a given gradient of pressure is greater (simply because the volume displacement that is required to generate a given gradient of pressure is greater), which also implies that the animal has to work harder for breathing. Again, these extra thoracic displacements are the ones required to decompress/compress air in the thorax and are thus out of phase with the nasal flow. So, an increased TGV will also increase the delay between the chambers and thereby increases sRaw. As can be seen, both bronchoconstriction and increased TGV result in a more important effort to draw air in-and-out of the lung. This is, in essence, the physiological meaning of sRaw. It represents the work required for breathing ${ }^{5,14}$.

It is thus important to understand that two distinct factors influence sRaw: airway resistance and TGV. In fact, sRaw can be expressed as the product of airway resistance and TGV ${ }^{11}$. Conscious animals can modify their TGV at will, as to adapt their ventilation to a given environment. Under such conditions, where the animal's natural physiological state is unaltered, it is thus impossible to discern whether a change in sRaw stems from an alteration in airway resistance, from a change in TGV, or from a mix of the two. Hence, it is recommended to complement the DCP evaluation with more invasive measurements of respiratory mechanics and/or lung volumes, such as those provided by the FOT ${ }^{1,15}$.

To date, the DCP has been used in various research applications. The technique can be used with or without the head chamber to quantitatively and accurately evaluate the effect of various substances, such as pharmaceutical agents, allergens, irritants or other mediators, on respiratory function in conscious small animals ${ }^{16,17,18}$. The front chamber can also be used as an exposing chamber to aerosolized substances or varying gas concentrations (hypoxia, hypercapnia, etc.) ${ }^{19}$. Conveniently, it allows one to concomitantly measure the acute effects of these exposures. In fact, one of the common uses of the DCP is to assess the degree of responsiveness to aerosolized methacholine in different models of respiratory diseases $\mathrm{s}^{20,21,22,23,24,25}$.

Although the DCP technique is seemingly straightforward, some practical challenges could potentially discourage inexperienced users or impair the accuracy and reproducibility of the results. The present paper provides a detailed description of the recommended procedures to successfully record respiratory function by DCP in conscious, restrained, spontaneously breathing mice. The description is specific to the stated equipment (please refer to the Table of Materials). The utility and value of the DCP is also demonstrated in a common model of pulmonary allergic inflammation in two strains of mice tested at baseline and in response to aerosolized methacholine.

\section{Protocol}

The following procedures were approved by the Quebec Heart and Lung Institute Animal Care Committee in accordance with the guidelines of the Canadian Council on Animal Care (CCAC).

\section{Preparation}

\section{Study}

1. (Critical) Prior to performing any experiment, obtain the appropriate approvals (e.g., IACUC) and trainings (e.g., animal handling).

2. Familiarize yourself with the equipment and the operating software. Read the user manuals and, if needed, create a configuration file to define the number of sites, input signals, analyzers, and parameters.

Note: Make sure to select a high sampling rate $(2 \mathrm{KHz})$.

3. Configure the analyzers settings for the parameters of interest.

1. Select Tuning from the tool bar and then Analyzers.

2. Adjust the flow threshold to a value that correctly separates the breaths (mouse: $0.5 \mathrm{~mL} / \mathrm{s}$ ) and opt for Ti + Te to compute the breathing rate.

3. Set a value for the barometric pressure $(760 \mathrm{~mm} \mathrm{Hg})$ and specify the maximal deviation of inspiratory/expiratory volume for a breath to be considered valid (mouse: $20 \%$ ).

4. Click on Setting in the Calculated parameters field to adjust the limits of acceptance.

Note: The following settings were used for the described mouse experiments: Inspiration time, 50 to 170 ms; expiration time, 40 to $180 \mathrm{~ms}$; frequency of breathing, 30 to $450 \mathrm{bpm}$; specific airway resistance, 0 to $15 \mathrm{cmH}_{2} \mathrm{O} \cdot \mathrm{s}$; midexpiratory flow with a precision of at least 3 decimals.

5. Once completed, select Apply and Close to exit the dialog window.

4. From the tool bar menu, go to Tuning again and then Storage to set a desired data storage rate. Click on Apply and Close to exit the window. 
Note: An average at every $10 \mathrm{~s}$ is typically used.

5. Create a protocol within the operating software to define a sequence of commands and the desired timing for each one. An example is displayed in Figure 1.

6. If the experiment involves the administration of a substance by aerosol, prepare the appropriate solutions and dilutions according to the concentrations to be tested.

\section{Animal}

1. Work in a quiet area remote from the housing room. Allow the animals to adjust to the change of environment.

2. Weigh the animals and select the appropriate restraint size.

3. (Critical) Acclimate the animals to the restrainer and procedures prior to the start of the experiment. Depending on the experimental design, multiple acclimation sessions of increasing duration (e.g., 5-30 min) may be needed.

Note: Animals that do not acclimate should be removed from the study.

1. At each acclimation session, insert the animal within the restrainer, proceeding from the back opening; holding the device vertically can be helpful.

2. Once the animal is in position, insert the back plunger and gently lock it in place without applying an excessive force.

3. (Critical) Visually check that the animal breathes normally. If needed, adjust its position by moving the locking mechanism. Make sure that the animal's nares are protruding outside of the nose-cone with its snout resting against the inner walls of the restrainer.

4. Detach the back panel of the thoracic chamber, insert the restrainer containing the animal through the rubber opening in the thoracic chamber, and close the chamber.

5. Attach the head chamber and provide a bias flow. Use a flow of $0.5 \mathrm{~L} / \mathrm{min}$ for a mouse.

6. Allow the animal to relax for 5 min.

7. Once the animal is calm, start the recording of the nasal and thoracic flow signals. Verify on the computer screen that the traces are smooth and that they display a regular breathing pattern; see example in Figure 2.

Note: If the protocol involves the aerosol administration of a substance, a saline challenge can be included in the acclimation procedure.

8. At the end of each session, remove the animal from the thoracic chamber and the restrainer and return it to its housing cage and room.

\section{Equipment}

1. On the day of the experiment, start an experimental session and load the appropriate configuration file.

Note: Verify that it contains the desired protocol for the experiment.

2. Go to Run in the tool bar menu. Enter the experiment and animal's information. Once done, click on the Run button at the bottom of the window.

3. Proceed with the calibration of the system. Calibrate each site and input signal separately.

1. Turn on the bias flow generator, connect it to the head chamber via a piece of tubing, and adjust the flow rate.

2. Close the top opening of the head chamber with a cap.

3. Detach the back panel of thoracic chamber, firmly insert the calibration tool inside the rubber opening between the head and the body chamber to create a hermetic seal. Then close and re-attach the back panel of the thoracic chamber

4. Verify that the side port of the thoracic chamber is capped.

5. From the software tool bar menu, go to Tuning and then Calibrate.

6. Go to Input 1 (thoracic) and select Calibrate to launch the calibration dialog box for the thoracic flow signal.

7. (Critical) Verify that the listed parameters in the calibration dialog window display the appropriate settings, i.e., Physical stress applied low value: 0; Physical stress applied high value: $-20 \mathrm{~mL} / \mathrm{s}$; Samples: Integrate. Once done, click on Low in the Samples window.

8. Verify that the signal generated is constant across the display window and then click on Close.

9. Connect a $20 \mathrm{~mL}$-syringe through the side port of the thoracic chamber using a plastic connector and a piece of tubing.

10. (Critical) Select High in the Samples window and immediately inject $20 \mathrm{~mL}$ of air into the chamber over a $2 \mathrm{~s}$ period at a flow rate as constant as possible.

11. Verify that the signal generated appears completely inside the display window. Use the arrow icon to verify whether the signal is centered and symmetric around the zero line. Then, click on Close. Remove any offset from zero by clicking on Remove AC offset in the Samples window Note: The high value calibration can be redone, if needed.

12. Verify that the resulting Scaled Input Range is within the recommended range (mouse: \pm 280 to $\pm 420 \mathrm{~mL} / \mathrm{s}$ ). (Critical) Repeat the calibration steps if the values are outside the acceptable range.

13. Calibrate the head chamber in a similar manner as the thoracic chamber (step 1.3.3.6). This time, select Input 2 (nasal). Note: (Important) The value for Physical stress applied high value must be set to $+20 \mathrm{~mL} / \mathrm{s}$. This will change the polarity of the flow in the front chamber in relation to the rear chamber. Hence, when the animal is breathing, the two flow signals will almost be in phase, aside from the delay used to calculate sRaw.

\section{Lung Function Measurements}

1. Weigh the animals and note their body weights.

2. Insert the animal within the restrainer and place it inside the thoracic plethysmograph chamber (Steps 1.2.3.1 to 1.2.3.5).

3. Allow the animal to relax for at least 5 min.

4. Start the protocol of commands by selecting the first step and then, click on Execute.

5. Check on the computer screen that the animal's breathing signals are regular and smooth (Figure 2). The software automatically displays the calculated parameters on a breath-by-breath basis. Verify that the animal's parameters are stable. 
6. Record the breathing pattern under baseline conditions for up to 10 minutes.

7. For protocols involving the administration of a test substance by aerosol, do as follows:

1. Adjust the nebulizer on-time and duty cycle, as required.

Note: In the examples demonstrated in this article, the nebulizer was operated at a $5 \%$ duty cycle for $10 \mathrm{~s}$.

2. Perform a vehicle challenge (e.g., saline) and record the response.

3. If needed, expose the animal to increasing concentrations of the test substance by changing the concentration in the nebulizer in escalating steps (e.g., doubling concentrations). Record the response after each administration.

4. At the end of the experimental session, if not done automatically, stop the recording and return the animal to its housing cage and room.

8. If needed, select Run from the tool bar menu to run another experimental session.

1. Between sessions, clean the plethysmograph chambers and rinse the nebulizer with water.

Note: The use of alcohol may cause irreversible damage to the plethysmograph.

9. If the study involves repeated evaluations over time, repeat the whole measurement sequence at each chosen timepoint Note: It is wisely recommended to complement the study with some precise measurements of respiratory mechanics and/or lung volumes ${ }^{1,15}$.

\section{Data Analysis}

Note: The software automatically saves the experimental file and exports the recorded parameters when the experimental session is closed.

1. Calculate a baseline average for the parameters of interest for each animal and experimental group.

Note: Table 1 lists a number of typical parameters, categorized based on the type of information they provide.

2. When relevant, evaluate the effect of the aerosolized substance studied on the parameters of interest at each concentration using a specific point (e.g., maximal or minimal value), an average, or the full time-course; a normalization to baseline can also be considered.

3. Report the results using the group means and errors in a table or graphic format. Analyze the results statistically.

Note: In the present study, two-way ANOVAs with repeated measures were used to assess the effect of methacholine, the allergen - housedust mite (HDM) - and their interaction on different DCP readouts (sRaw and EF50), as well as on different FOT readouts ( $R_{N}, G$ and $H$ ), in both strains of mice. Sidak's multiple comparison tests were then used to determine the concentrations of methacholine at which the allergic mice differ from the control mice. The same tests were used to assess the effect of days, HDM and their interaction at baseline (i.e., before methacholine) on readouts of airway obstruction (sRaw and EF50) and of ventilation pattern (BF, TV, MV and EIP). Pearson's correlations were used to evaluate the correlations between sRaw obtained with the DCP and $R_{N}$ obtained with the FOT. All statistical analyses and graphs were performed using alternative standard statistical software (e.g., GraphPad Prism). p $\leq 0.05$ was considered sufficient to reject the null hypothesis.

\section{Representative Results}

The results of repeated evaluations of respiratory function by DCP, performed under baseline conditions on three consecutive days (days 12 , 13 , and 14 of the protocol illustrated in Figure 1) in control and allergic BALB/c mice, are shown in Figure 3 . The parameters that were selected to assess the pattern of breathing included breathing frequency (Figure 3A), tidal volume (Figure 3B), minute ventilation (Figure 3C), and end-inspiratory pause (Figure 3D). The parameters used to assess airway obstruction were EF50 (Figure 3E) and sRaw (Figure 3F). The outcomes of each selected parameter were stable over these three consecutive days in both groups, with no apparent effect caused by allergic inflammation.

The degree of responsiveness to methacholine was evaluated by DCP on successive days (days 12, 13, and 14 of the protocol illustrated in Figure 1) in both control and allergic BALB/c mice. The results, shown in Figure 4, display the changes in the two parameters that are sensitive to airway obstruction, namely sRaw (Figure 4A, B and C) and EF50 (Figure 4D, E and F). As expected, incremental concentrations of methacholine progressively increased sRaw and progressively decreased EF50. These responses were potentiated by allergic inflammation, especially at the final concentration tested, which attested the presence of hyperresponsiveness. The results also demonstrate that the exaggerated degree of responsiveness was confined to the first day (day 12), as it was not observed during the two subsequent assessments (i.e., at days 13 and 14 ).

The results of the assessment of respiratory mechanics by the FOT, performed on the last day of the experimental protocol (day 15 ; Figure 1) in both control and allergic BALB/c mice, are shown in Figure 5. These experiments were included in the study to complement the DCP evaluations. The FOT is recognized as a more precise approach to assess respiratory function ${ }^{2}$. One of its strengths is that it provides topographic insights as to which sites of the lung (conducting airways versus peripheral airways and lung tissue) are affected by the tested interventions (e.g., allergen and methacholine). The recommended methodology to assess respiratory mechanics with the FOT was previously described ${ }^{1}$. Herein, three FOT parameters were used to describe the changes in respiratory mechanics induced by allergic inflammation and methacholine. These parameters included: 1-Newtonian resistance $\left(R_{N}\right.$; Figure $\left.\mathbf{5 A}\right)$, a parameter for which the changes in value mainly reflect variations in the resistance of the large conducting airways; 2-tissue damping (G; Figure 5B), a parameter for which the changes in value mainly reflect variations in tissue resistance; and 3-tissue elastance $(\mathrm{H}$; Figure $\mathbf{5 C})$, a parameter for which the changes in value mainly reflect variations in tissue stiffness ${ }^{2}$. As expected, there was an increase in each of these parameters in response to incremental concentrations of methacholine. Consistent with sRaw and EF50 results obtained with the DCP from the previous day (day 14; Figure 1), the changes in $R_{N}$ induced by methacholine (Figure 5A) were comparable between the control and the allergic mice. In fact, the values of $s$ Raw at day 14 correlated with the values of $R_{N}$ at day 15 (Figure $5 \mathrm{D}$ ). The rise in $\mathrm{H}$ induced by methacholine was also similar between the control and the allergic mice (Figure 5B). However, the methacholine-induced increase in $\mathrm{G}$ was significantly greater in the allergic mice (Figure 5C). This result demonstrates the presence of a persisting hyperresponsive phenotype in the allergic mice at day 15 , which was not detected by the DCP evaluations performed on the two previous days. 
The entire study was repeated with C57BL/6 mice. The results of the successive DCP evaluations of sRaw, at days 12,13 , and 14 of the protocol (Figure 1), and of the FOT evaluation of $R_{N}$, at day 15, are shown in Figure 6. In that specific mouse strain, the exaggerated methacholine response observed in the allergic mice was maintained over the three consecutive days (Figure 6A, B, and C). This hyperresponsive phenotype was also depicted with the FOT at day 15 by a rise in $R_{N}$ induced by methacholine that was more pronounced in the allergic mice (Figure 6E). These were in stark contrast with the results obtained with the BALB/c mice, where a progressive waning of the hyperresponsiveness occurred from days 12 to 14 (Figure 4) and a lack of difference in the methacholine-induced rise in $R_{N}$ was observed at day 15 (Figure 5A). Together, these results indicated a time-varying effect of the allergen on the methacholine-induced response between the two strains of mice. Importantly, this strain difference was depicted by both the DCP and the FOT. Concordantly, the values of sRaw measured by the DCP at day 14 correlated with the values of $R_{N}$ measured by FOT at day 15 (Figure 6F), as was observed with the BALB/c mice (Figure 5D).

A

Saline or HDM

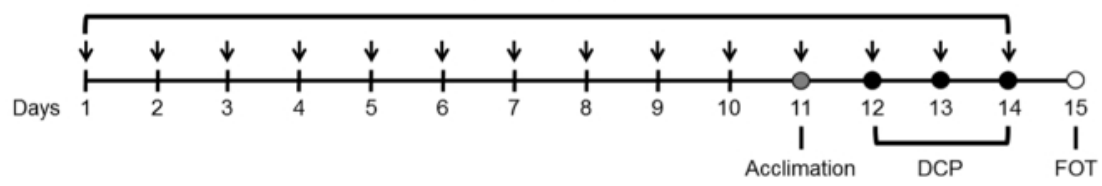

B

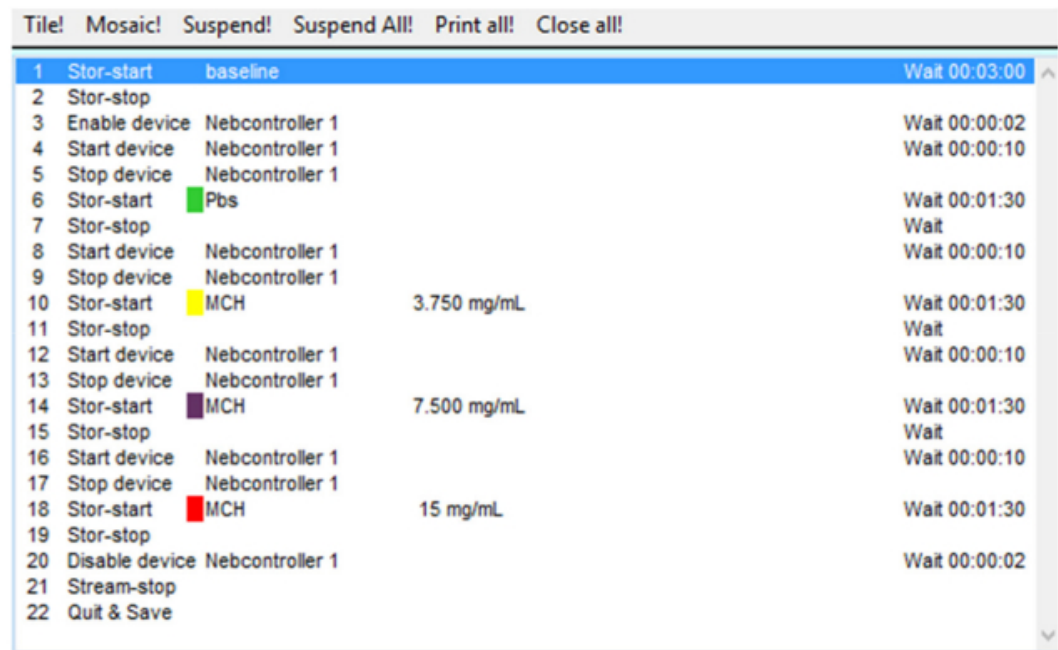

Figure 1. Protocols used to induce pulmonary allergic inflammation and to assess the degree of responsiveness to methacholine. This study was conducted on female BALB/c and C57BL/6 mice of 7 to 9 weeks of age. The sequence of interventions carried out over the entire study is shown in panel (A). Half of the mice was exposed to $50 \mu \mathrm{g}$ of house-dust mite (HDM) extract intranasally on 14 consecutive days to induce pulmonary allergic inflammation. The other half was exposed to saline and used as control. Respiratory function was assessed by double-chamber plethysmography (DCP) on three separate occasions (Days 12, 13, and 14; black circles) following an acclimation session (Day 11; gray circle) that included a challenge with aerosolized saline. During each session, baseline respiratory function and the response to methacholine were assessed using the automated protocol shown in panel (B). At day 15, an invasive assessment of respiratory mechanics with the forced oscillation technique (FOT) was performed as previously described ${ }^{1}$. Please click here to view a larger version of this figure. 


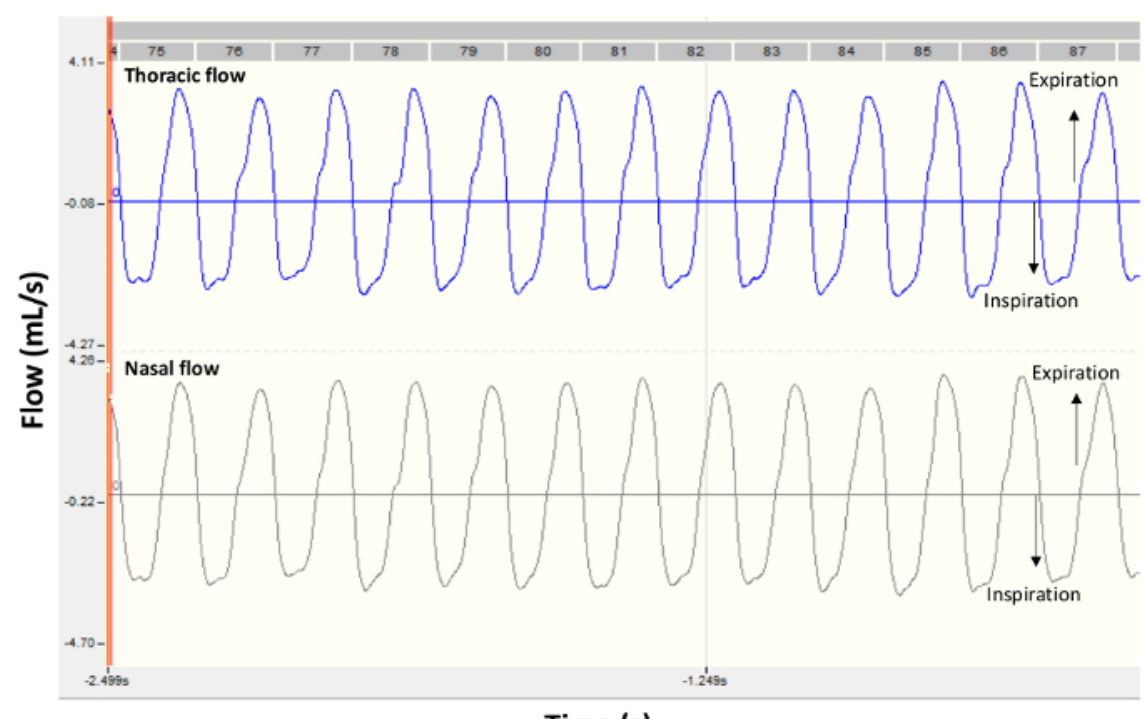

Time (s)

Figure 2. Representative flow signals from a healthy BALB/c mouse. The panels show typical recording traces obtained by double-chamber plethysmography in a control mouse under baseline conditions. Thoracic flow is shown in the upper panel and nasal flow is shown in the lower panel. Negative values are during inspiration and positive values are during expiration. Please click here to view a larger version of this figure.

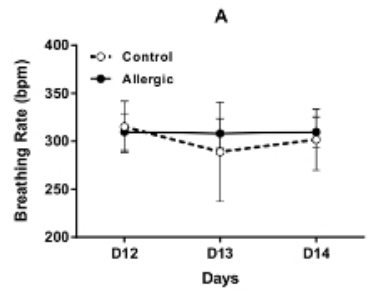

D

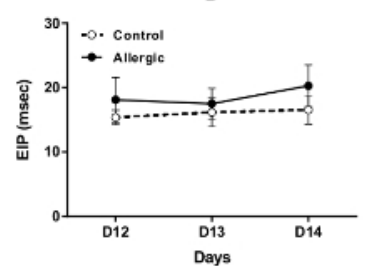

B

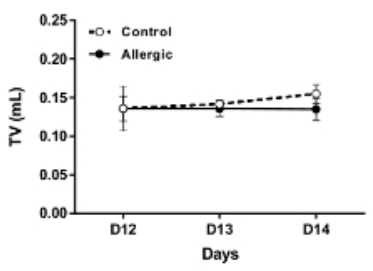

E

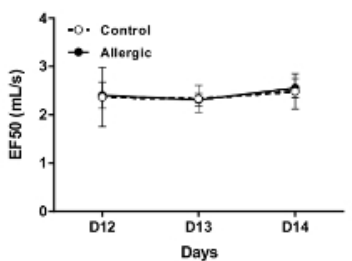

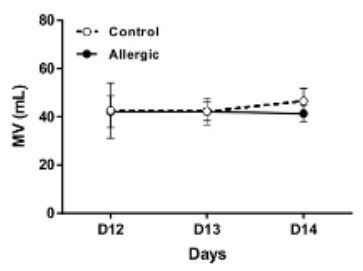

F

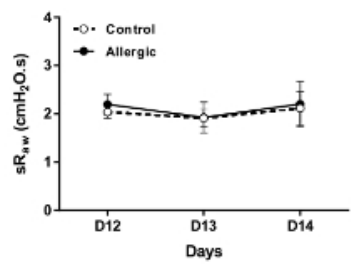

Figure 3. Repeated evaluations of respiratory function in conscious BALB/c mice. Baseline respiratory function was assessed by double-chamber plethysmography (DCP) in control (open symbols) and allergic (solid symbols) mice on days 12, 13, and 14 of the protocol illustrated in Figure 1. The DCP parameters used to assess respiratory function included breathing frequency in (A), tidal volume (TV) in $(B)$, minute ventilation (MV) in (C), end inspiratory pause (EIP) in (D), flow at mid-tidal expiratory volume (EF50) in (E), and specific airway resistance (sRaw) in (F). The values of breathing frequency, TV, MV, sRaw and EIP for each mouse were the average values recorded over $1.5 \mathrm{~min}$. The value of EF50 was the minimal value obtained during this recording period. The results are presented as group means \pm standard deviation ( $n=5 /$ group). Please click here to view a larger version of this figure. 
Day 12

A

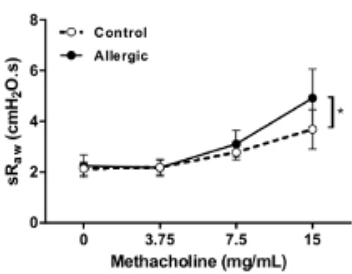

D

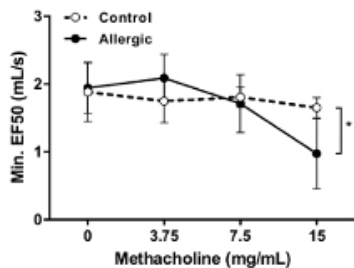

Day 13
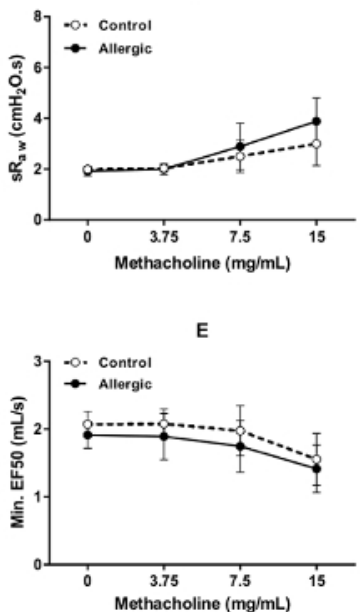

Day 14

C
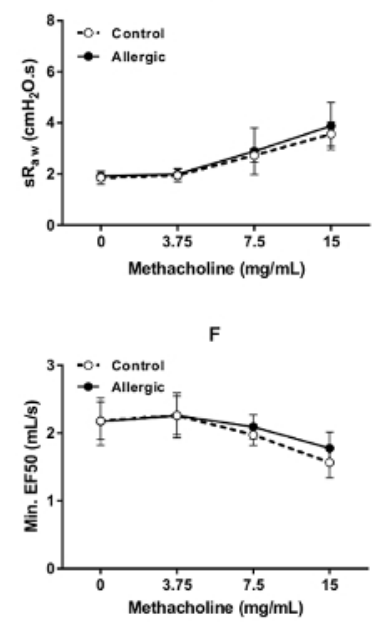

Figure 4. Methacholine bronchoprovocation test in conscious BALB/c mice. Methacholine responsiveness was assessed by doublechamber plethysmography (DCP) in control (open symbols) and allergic (solid symbols) mice on days 12, 13, and 14 of the protocol illustrated in Figure 1. The DCP parameters used to assess the response included specific airway resistance (sRaw) in (A) through (C) and flow at mid-tidal expiratory volume (EF50) in (D) through (F). The bronchoprovocation was performed by aerosolizing methacholine in the DCP head chamber for $10 \mathrm{~s}$ at incremental concentrations. The response was monitored during $1.5 \mathrm{~min}$ after each concentration. The value of sRaw for each mouse at each concentration was the average value recorded over $1.5 \mathrm{~min}$. The value of EF50 was the minimal value obtained during this recording period. The results are presented as group means \pm standard deviation ( $n=5 / g r o u p)$. The asterisk symbol * designates a statistically significant difference $(p \leqq 0.05)$. Please click here to view a larger version of this figure.

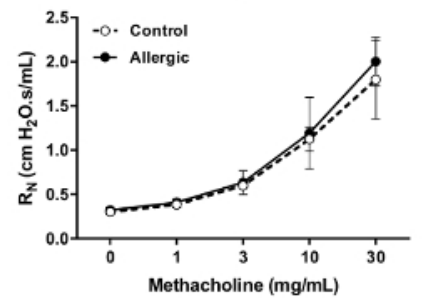

C

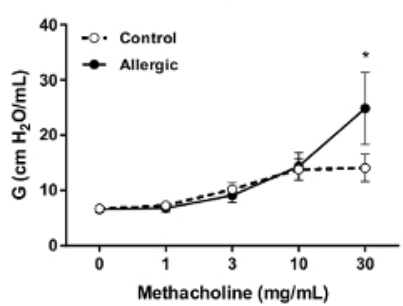

B

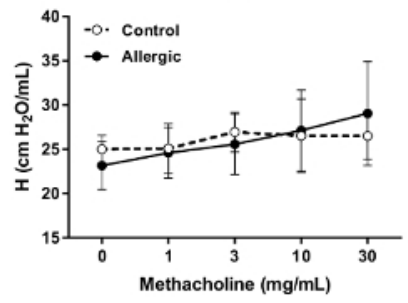

D

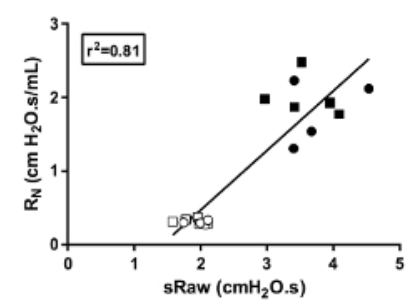

Control - Baseline

- Control - Methacholine

- Allergic - Baseline

- Allergic - Methacholin

Figure 5. Invasive assessment of respiratory mechanics in BALB/c mice. Respiratory mechanics at baseline and in response to methacholine was evaluated by the forced oscillation technique (FOT) on Day 15 of the protocol illustrated in Figure 1. The control (open symbols) and the allergic (solid symbols) mice were the same as the ones tested by double-chamber plethysmography (DCP) on Days 12 , 13 , and 14. The parameters used to assess respiratory mechanics were Newtonian resistance $\left(R_{N)}\right.$ in $(A)$, tissue elastance $(H)$ in $(B)$ and tissue damping $(\mathrm{G})$ in $(\mathbf{C})$. The bronchoprovocation was performed by nebulizing incremental concentrations of methacholine directly into the endotracheal tube of anesthetized, tracheotomized, paralyzed, and mechanically ventilated mice in supine position. The response was monitored during $5 \mathrm{~min}$ after each concentration. The value for each parameter for each mouse at each concentration was the peak value obtained during this recording period. The results are presented as group means \pm standard deviation ( $n=5 /$ group). Panel (D) shows the correlation between specific airway resistance (sRaw) measured by DCP on day 14 and $R_{N}$ measured by FOT on day 15 . The open symbols represent the values at baseline and the solid symbols represent the maximal values at the highest concentration of methacholine tested for either the control (circles) or the allergic (squares) mice. The inset shows the coefficient of determination $\left(r^{2}\right)$. The asterisk symbol * designates a statistically significant difference $(p \leq 0.05)$. Please click here to view a larger version of this figure. 

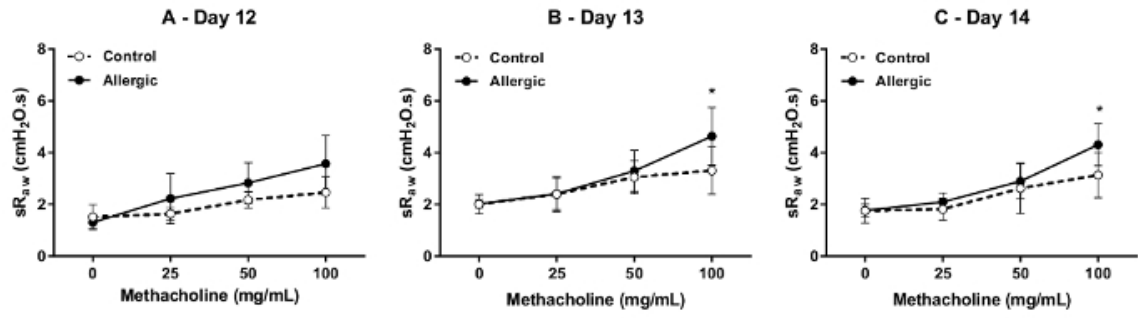

D. Day 15
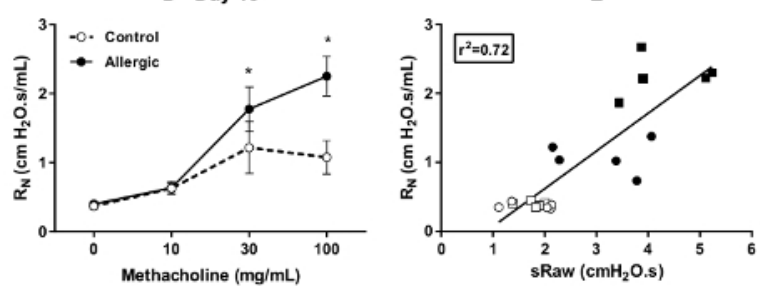

- Control - Baseline

- Control - Methacholin

a Allorgic - Baselino

- Allergic - Methacholine

Figure 6. Respiratory function and invasive respiratory mechanics in C57BL/6 mice. Specific airway resistance (sRaw) was assessed by double-chamber plethysmography (DCP) at baseline and in response to methacholine in control (open symbols) and allergic (solid symbols) mice on days $12(\mathbf{A}), 13(\mathbf{B})$, and $14(\mathbf{C})$ of the protocol illustrated in Figure 1. Newtonian resistance $\left(R_{N}\right)$ at baseline and in response to methacholine were assessed by the forced oscillation technique (FOT) on day 15 (D). The bronchoprovocations were performed as described in Figure 4 and Figure 5 for the DCP and the FOT, respectively. The results are presented as group means \pm standard deviation ( $\mathrm{n}=5 / \mathrm{group}$ ). Panel (E) shows the correlation between sRaw measured by DCP on day 14 and $R_{N}$ measured by FOT on day 15 . The open symbols represent the values at baseline and the solid symbols represent the maximal values at the highest concentration of methacholine tested for either the control (circles) or the allergic (squares) mice. The inset shows the coefficient of determination $\left(r^{2}\right)$. The asterisk symbol * designates a statistically significant difference $(p \leq 0.05)$. Please click here to view a larger version of this figure.

\begin{tabular}{|c|c|c|c|}
\hline Parameter & Unit & Description & Information \\
\hline $\mathrm{F}$ & bpm & Breathing frequency & \multirow[t]{12}{*}{ Pattern of ventilation } \\
\hline TV & $\overline{\mathrm{mL}}$ & Tidal volume & \\
\hline MV & $\mathrm{mL}$ & Minute ventilation & \\
\hline $\mathrm{Ti}$ & $\mathrm{ms}$ & Inspiratory time & \\
\hline $\mathrm{Te}$ & $\mathrm{ms}$ & Expiratory time & \\
\hline PIF & $\mathrm{mL} / \mathrm{s}$ & Peak inspiratory flow & \\
\hline PEF & $\mathrm{mL} / \mathrm{s}$ & Peak expiratory flow & \\
\hline EV & $\mathrm{mL}$ & Expiratory volume & \\
\hline NTV & $\mathrm{mL}$ & Nasal tidal volume & \\
\hline NEV & $\mathrm{mL}$ & Nasal expiratory volume & \\
\hline EIP & $\mathrm{ms}$ & End inspiratory pause & \\
\hline EEP & $\mathrm{ms}$ & End expiratory pause & \\
\hline $\mathrm{dT}$ & $\mathrm{ms}$ & Time delay & \multirow[t]{4}{*}{ Airflow obstruction } \\
\hline sRaw & $\mathrm{cmH}_{2} \mathrm{O} \cdot \mathrm{s}$ & Specific airway resistance & \\
\hline sGaw & $1 / \mathrm{cmH}_{2} \mathrm{O} \cdot \mathrm{s}$ & Specific airway conductance & \\
\hline EF50 & $\mathrm{mL} / \mathrm{s}$ & Flow at mid-tidal expiratory volume & \\
\hline $\mathrm{Sr}$ & $\%$ & Success rate & \multirow[t]{2}{*}{ Quality control } \\
\hline $\mathrm{N}$ & & Number of valid breaths & \\
\hline
\end{tabular}

Table 1. List of typical parameters obtained from double-chamber plethysmography. The parameters were grouped according to the nature of the information they provide during a respiratory function evaluation. 


\begin{tabular}{|l|l|}
\hline Advantages & Limitations \\
\hline$\cdot$ Conscious animals & - Necessity to control the surrounding environment \\
\hline$\cdot$ Accurate ventilation parameters & - Prior acclimation of the animals \\
\hline$\cdot$ Undisputed indexes of airflow obstruction (sRaw, EF50) & $\cdot$ Requirement to hermetically separate the nasal and thoracic flows \\
\hline$\cdot$ Adaptable to various species and animal sizes & - Absolute value variability for some outcome parameters \\
\hline$\cdot$ Used in many research applications & - sRaw not a true measurement of resistance \\
\hline - Straightforward technique & - Presence of the upper airways \\
\hline - Sensitive to change & - Complementing the measurements with an invasive assessment \\
\hline
\end{tabular}

Table 2. List of advantages and limitations associated with double-chamber plethysmography.

\begin{tabular}{|c|c|c|}
\hline & Double-Chamber Plethysmography & Forced Oscillation Technique \\
\hline Animal's state of consciousness & Unaltered & Anesthetized (and usually paralyzed) \\
\hline Animal's position & Upright & Supine \\
\hline Animal's accessibility & Confined within chamber & Accessible \\
\hline $\begin{array}{l}\text { Animal integration to the measurement } \\
\text { device }\end{array}$ & Nose or neck seal & Tracheotomy or oral intubation \\
\hline Animal's airway tree & Intact & $\begin{array}{l}\text { Partial - upper airway segment excluded (i.e. } \\
\text { nasal conducts, pharynx and larynx) }\end{array}$ \\
\hline $\begin{array}{l}\text { Lung volume at which the outcome } \\
\text { parameters are obtained }\end{array}$ & $\begin{array}{l}\text { Variable - spontaneous volume adopted by the } \\
\text { animal }\end{array}$ & $\begin{array}{l}\text { Standardized - using controlled recruitment } \\
\text { maneuvers and positive end-expiratory } \\
\text { pressure. }\end{array}$ \\
\hline $\begin{array}{l}\text { Frequency at which the outcome } \\
\text { parameters are assessed }\end{array}$ & $\begin{array}{l}\text { Variable - spontaneous breathing frequency } \\
\text { adopted by the animal }\end{array}$ & $\begin{array}{l}\text { Controlled - using predefined waveforms at } \\
\text { specified frequencies }\end{array}$ \\
\hline $\begin{array}{l}\text { Contribution from upper airway segment to } \\
\text { the outcome parameters }\end{array}$ & To be expected & Circumvented \\
\hline Site of aerosol delivery & Inside the head chamber & Directly in the trachea \\
\hline $\begin{array}{l}\text { Effect of upper airway segment on inhaled } \\
\text { dose/ aerosol deposition pattern }\end{array}$ & To be expected & Prevented \\
\hline $\begin{array}{l}\text { Ability to detect change - based on the } \\
\text { results of the present study }\end{array}$ & Observed & Observed \\
\hline $\begin{array}{l}\text { Inherent variability of the technique - based } \\
\text { on the results of the present study }\end{array}$ & $\begin{array}{l}\text { Fluctuation of the coefficient of variation for } \\
\text { sRaw at baseline: } 7.5-20.6 \%\end{array}$ & $\begin{array}{l}\text { Fluctuation of the coefficient of variation for } R_{N} \\
\text { at baseline: } 3.6-13.4 \%\end{array}$ \\
\hline
\end{tabular}

Table 3. Comparison between the double-chamber plethysmography and forced oscillation techniques.

\section{Discussion}

The ability to measure lung function in conscious animals is clearly warranted in respiratory research. In general, the DCP is an interesting approach to evaluate the ventilation function of the respiratory system in conscious and spontaneously breathing animals ${ }^{26}$. More specifically, the $\mathrm{DCP}$, or its head-out variant, often strikes a right balance between the quality of the information provided and the desired level of invasiveness ${ }^{3}$ (Table 2). The technique can be adapted to various species (e.g., mouse, rat, guinea pig) or animal sizes and can be used in many research applications. It is particularly useful to assess numerous animals at once in a parallel study design, to monitor the respiratory function in repeated manner, and to capture the kinetics of a response over time. In addition, the technique is straightforward and can be learned in a relatively timely manner. In the present paper, a protocol employing DCP measurements in mice was used as an example to describe the hands-on aspects of this restrained plethysmography technique as well as to discuss the critical steps and related outcomes.

When working with conscious animals, it is essential to control the conditions of the surrounding environment (e.g., quiet room with a limited number of people or activity) in order to generate reproducible results. Since the restrainers come in various dimensions, it is important to start with the appropriate size so that the breathing movements are unperturbed. It is also helpful and often required to acclimate the animals to the experimental set-up and procedures, as it is well established in mice that restraining affects the breathing frequency ${ }^{12}$. Depending on the experimental design or conditions, multiple sessions of incremental durations may be needed. Finally, allowing time at the start of an experiment for the animals to adjust to the room change and necessary handling is a simple consideration that proved effective to ensure that the breathing pattern is consistently regular and relaxed at baseline. Working under conditions where the animals are comfortable, well-adapted, and calm will also be beneficial in terms of result variability and quality. It also limits any stress-induced release of catecholamine, which may increase airway caliber and attenuate an induced bronchoconstriction.

It is important to understand that there is a need to separate as hermetically as possible the nasal and thoracic flows. Depending on the system or species studied, the sealing mechanism can vary in shape as well as in efficacy. In the DCP we described herein, the seal is created between 
the animal's snout and the restraining device. When assessing the respiratory function by $\mathrm{DCP}$, it is also essential to provide a sufficient and continuous bias flow, as a decrease in the level of oxygen available to the animal will result in significant effects. Taking into account the wellbeing of the animal in the restrainer limits the propensity for air leaks created by agitation and thereby maximizes the quality of the data. Contrastingly, a breaking in the seal will result in either rejected datasets or an underestimation of some parameters.

In addition to enable the separate recording of the nasal flow signal, the head chamber is typically used to expose the animal to aerosolized substances. As illustrated in this article, this can be utilized to perform a bronchoprovocation test to demonstrate differing degrees of responsiveness. In such experiments, adjusting the range of tested concentrations may be needed depending on the species, strain, or sex of the animals studied. As previously demonstrated ${ }^{8,9,10,27}$, the present results show that the methacholine-induced changes in sRaw correlated well with invasive FOT measurements of airway resistance. The results also demonstrate that the DCP technique is not as sensitive as the FOT for its ability to detect respiratory dysfunction and to identify an altered response localized within the lower compartments of the lung (lung tissue and/or small peripheral airways). Since the animal's airways are intact, the presence of the upper airways, which accounts for the largest part of the total respiratory resistance to airflow ${ }^{28}$, can affect the aerosol distribution and deposition in addition to dampen the contribution from the lower airways to a measurement. Table 3 summarizes other differences between the DCP technique and the FOT. Finally, while it would be theoretically possible to estimate the animal's total airway resistance (including upper airways) from a measurement of sRaw, it is generally recommended to complement the DCP evaluation with an invasive measurement technique such as the FOT ${ }^{29}$ to obtain direct measurements of detailed respiratory mechanics. Depending on the objectives of the study, measurements of upper airway resistance can also be considered ${ }^{30,31,32}$

\section{Conclusion}

Owing to its limited degree of invasiveness, the DCP is a technique that can fulfill an important need in respiratory research. It is able to provide accurate readouts of ventilation pattern in conscious animals concurrently with some undisputed indexes of airflow obstruction. The information obtained also truly complements that from more invasive approaches.

\section{Disclosures}

DB and AR are employed by SCIREQ Scientific Respiratory Equipment Inc, a commercial entity involved in topics related to the content of this article. DB also owns stock. SCIREQ Inc. is an emka TECHNOLOGIES company.

\section{Acknowledgements}

SML is supported by a studentship from the Canadian Institutes of Health Research, MG is supported by a bursary from the Respiratory Health Network of the FRQS (Fonds de recherche du Québec - Santé) and YB is a research scholar from FRQS.

\section{AUTHORS' CONTRIBUTION}

All authors contributed to the conception of the manuscript and/or the video. SML and LD collected the data. SML, LD, YB, DM, DB and AR contributed to data analysis, the generation of figures and manuscript writing. $Y B, A R, K L$ and $M G$ were involved in preparing the video script. The play was performed by $\mathrm{YB}, \mathrm{KL}$ and MG.

\section{References}

1. McGovern, T. K., Robichaud, A., Fereydoonzad, L., Schuessler, T. F., \& Martin, J. G. Evaluation of respiratory system mechanics in mice using the forced oscillation technique. J Vis Exp. (75), e50172 (2013).

2. Bates, J. H. T. CORP: Measurement of lung function in small animals. J Appl Physiol (1985). 123 (5), 1039-1046 (2017).

3. Bates, J. H., \& Irvin, C. G. Measuring lung function in mice: the phenotyping uncertainty principle. J Appl Physiol. 94 (4), 1297-1306 (2003).

4. Lim, R. et al. Measuring respiratory function in mice using unrestrained whole-body plethysmography. $J$ Vis Exp. (90), e51755 (2014).

5. Enhorning, G., van Schaik, S., Lundgren, C., \& Vargas, I. Whole-body plethysmography, does it measure tidal volume of small animals? Can J Physiol Pharmacol. 76 (10-11), 945-951 (1998).

6. Vijayaraghavan, R., Schaper, M., Thompson, R., Stock, M. F., \& Alarie, Y. Characteristic modifications of the breathing pattern of mice to evaluate the effects of airborne chemicals on the respiratory tract. Arch Toxicol. 67 (7), 478-490 (1993).

7. Willis, D. N., Liu, B., Ha, M. A., Jordt, S. E., \& Morris, J. B. Menthol attenuates respiratory irritation responses to multiple cigarette smoke irritants. FASEB J. 25 (12), 4434-4444 (2011).

8. Neuhaus-Steinmetz, U. et al. Sequential development of airway hyperresponsiveness and acute airway obstruction in a mouse model of allergic inflammation. Int Arch Allergy Immunol. 121 (1), 57-67 (2000).

9. Glaab, T. et al. Tidal midexpiratory flow as a measure of airway hyperresponsiveness in allergic mice. Am J Physiol Lung Cell Mol Physiol. 280 (3), L565-573 (2001).

10. Glaab, T. et al. Noninvasive measurement of midexpiratory flow indicates bronchoconstriction in allergic rats. J Appl Physiol (1985). 93 (4), 1208-1214 (2002).

11. Pennock, B. E., Cox, C. P., Rogers, R. M., Cain, W. A., \& Wells, J. H. A noninvasive technique for measurement of changes in specific airway resistance. J Appl Physiol Respir Environ Exerc Physiol. 46 (2), 399-406 (1979).

12. DeLorme, M. P., \& Moss, O. R. Pulmonary function assessment by whole-body plethysmography in restrained versus unrestrained mice. $J$ Pharmacol Toxicol Methods. 47 (1), 1-10 (2002).

13. Flandre, T. D., Leroy, P. L., \& Desmecht, D. J. Effect of somatic growth, strain, and sex on double-chamber plethysmographic respiratory function values in healthy mice. J Appl Physiol (1985). 94 (3), 1129-1136 (2003).

14. Criee, C. P. et al. Body plethysmography--its principles and clinical use. Respir Med. 105 (7), 959-971 (2011).

15. Robichaud, A. et al. Automated full-range pressure-volume curves in mice and rats. J Appl Physiol (1985). 123 (4), 746-756 (2017). 
16. Mizutani, N., Goshima, H., Nabe, T., \& Yoshino, S. Complement C3a-induced IL-17 plays a critical role in an IgE-mediated late-phase asthmatic response and airway hyperresponsiveness via neutrophilic inflammation in mice. J Immunol. 188 (11), $5694-5705$ (2012).

17. Nabe, T. et al. Roles of basophils and mast cells infiltrating the lung by multiple antigen challenges in asthmatic responses of mice. $\mathrm{Br} J$ Pharmacol. 169 (2), 462-476 (2013).

18. Morris, J. B. et al. Immediate sensory nerve-mediated respiratory responses to irritants in healthy and allergic airway-diseased mice. J Appl Physiol (1985). 94 (4), 1563-1571 (2003).

19. Merazzi, D., \& Mortola, J. P. Effects of changes in ambient temperature on the Hering-Breuer reflex of the conscious newborn rat. Pediatr Res. 45 (3), 370-376 (1999).

20. Rao, R., Nagarkatti, P. S., \& Nagarkatti, M. Delta(9) Tetrahydrocannabinol attenuates Staphylococcal enterotoxin B-induced inflammatory lung injury and prevents mortality in mice by modulation of miR-17-92 cluster and induction of T-regulatory cells. $\mathrm{Br} \mathrm{J} P$ harmacol. 172 (7), 1792-1806 (2015).

21. Agrawal, A. et al. Inhibition of mucin secretion with MARCKS-related peptide improves airway obstruction in a mouse model of asthma. $J$ Appl Physiol (1985). 102 (1), 399-405 (2007).

22. Mabalirajan, U., Aich, J., Agrawal, A., \& Ghosh, B. Mepacrine inhibits subepithelial fibrosis by reducing the expression of arginase and TGFbeta1 in an extended subacute mouse model of allergic asthma. Am J Physiol Lung Cell Mol Physiol. 297 (3), L411-419 (2009).

23. Desmet, C. et al. Treatment of experimental asthma by decoy-mediated local inhibition of activator protein-1. Am J Respir Crit Care Med. 172 (6), 671-678 (2005).

24. Zang, N. et al. Pulmonary C Fibers Modulate MMP-12 Production via PAR2 and Are Involved in the Long-Term Airway Inflammation and Airway Hyperresponsiveness Induced by Respiratory Syncytial Virus Infection. J Virol. 90 (5), 2536-2543 (2015).

25. Shukla, M. et al. Carryover of cigarette smoke effects on hematopoietic cytokines to F1 mouse litters. Mol Immunol. 48 (15-16), 1809-1817 (2011).

26. Murphy, D. J. Respiratory function assessment in safety pharmacology. Curr Protoc Pharmacol. Chapter 10 Unit10 19 (2003).

27. Lofgren, J. L. et al. Restrained whole body plethysmography for measure of strain-specific and allergen-induced airway responsiveness in conscious mice. J Appl Physiol (1985). 101 (5), 1495-1505 (2006).

28. DiMaria, G. U., Wang, C. G., Bates, J. H., Guttmann, R., \& Martin, J. G. Partitioning of airway responses to inhaled methacholine in the rat. J Appl Physiol (1985). 62 (3), 1317-1323 (1987).

29. Hoymann, H. G. Lung function measurements in rodents in safety pharmacology studies. Front Pharmacol. 3 156 (2012).

30. Agrawal, A., Singh, S. K., Singh, V. P., Murphy, E., \& Parikh, I. Partitioning of nasal and pulmonary resistance changes during noninvasive plethysmography in mice. J Appl Physiol (1985). 105 (6), 1975-1979 (2008).

31. McLeod, R. L., Young, S. S., Erickson, C. H., Parra, L. E., \& Hey, J. A. Characterization of nasal obstruction in the allergic guinea pig using the forced oscillation method. J Pharmacol Toxicol Methods. 48 (3), 153-159 (2002).

32. Miyahara, S., Miyahara, N., Takeda, K., Joetham, A., \& Gelfand, E. W. Physiologic assessment of allergic rhinitis in mice: role of the highaffinity IgE receptor (FcepsilonRI). J Allergy Clin Immunol. 116 (5), 1020-1027 (2005). 